\title{
The innocence of Omicron: Taking the right decision
}

\section{Ruby Dhar ${ }^{1}$, Arun Kumar ${ }^{2}$, Subhradip Karmakar ${ }^{3}$}

${ }^{1}$ Scientist, ${ }^{3}$ Additional Professor, Department of Biochemistry, All India Institute of Medical Sciences, New Delhi, ${ }^{2}$ Professor, Department of Biochemistry, Jagannath Gupta Institute of Medical Sciences and Hospital, Kolkata, West Bengal, India

Rapidly spreading Omicron (B1.1.529) poses a considerable health risk, despite being labeled as a mild SARS-CoV2 variant. The higher transmissibility, immune evasion properties along blunting the effect of vaccine are some of its unique features, a fact that should not be ignored, no matter how benign it might be. We suggest that our knowledge and experience in dealing with Omicron have been very less and its long-term health consequences are still not studied properly. Under these conditions, we must continue to remain vigilant following all safety measures and avoid down-sizing its impact.

Key words: COVID19; Immunity; Omicron; Pandemic; Variant
http://nepjol.info/index.php/AJMS DOI: 10.3126/ajms.v13i3.43020

E-ISSN: 2091-0576

P-ISSN: 2467-9100

Copyright (c) 2022 Asian Journal of Medical Sciences

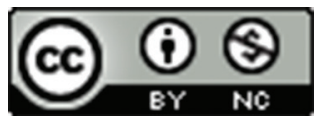

This work is licensed under a Creative Commons Attribution-NonCommercial 4.0 International License.

\section{INTRODUCTION}

Omicron (B1.1.529) quickly evolved to become a VoC within a few months' time, spreading from South Africa to several nations worldwide. ${ }^{1}$ The Technical Advisory Group on SARS-CoV-2 virus evolution monitoring the evolution of SARS-CoV-2 was convened to assess this variant after it was first reported to the WHO from South Africa on November 24, 2021 from a specimen collected on November 9, 2021. The detection of B1.1.529 quickly coincided with a surge in COVID-19 cases establishing it as the dominant variant. By the time travel advisories and other restrictions could kick in, Omicron already spread to 89 countries (as of $18^{\text {th }}$ Dec 2021) infecting millions. According to the Institute for Health Metrics and Evaluation, Washington, Omicron infections are set to surge in the next $2-3$ months and may infect $1-2$ billion people globally. What makes B1.1.529 special is the sheer number of mutations it accumulated (32 vs. 12) as compared to the delta (B1.617.2), plus the rapidness it is spreading with an estimated Ro of 2.69 (vs. 1.69 for the delta variant). For a populous country like India with over 8 lakh active cases $^{2}$ and a positivity of $\sim 15-20 \%$, the stakes are higher. The health-care system that is already stretched beyond its capacity has seen a further dent with several HCW exposed and quarantined. Amidst the rising cases, the administration has to enforce lockdown, containment zones, and travel restrictions which may, in turn, affect the economy. However, it seems during this crisis, the public is getting influenced by a different set of narratives, derived from an overall short term observation, poor understanding of the full nature and scale of this variant, inadequate testing, surveillance, and genome sequencing, as well as overlooking the long-term health consequences of Omicron on adults as well as in children and pregnant women. This lack of our knowledge 
is giving rise to numerous misleading statements ${ }^{3}$ even by the professionals, labeling Omicron to be a mild, benign variant, a variant of least concern, a variant that needs nearly no hospitalization or is associated with very low mortality. Taken to the public domain, this may create callousness and a sense of anarchy in following COVID-appropriate behavior such as social distancing, double masking, or getting tested and vaccinated. This also falsely justifies hosting super-spreader events such as religious and political rallies, ignoring the law and order, and downsizing the potential risk. We need to accept from all our past experiences that our understanding of the overall COVID pandemic is still very naïve and almost nil for this new variant. Still, a vast majority of the PCR/NAAT-based detection is not targeted to B1.1.529. $\mathrm{S}$ Gene target failure is rarely used in diagnostic laboratories. With India's sequencing agency, The Indian SARS-CoV-2 Genomics Consortium, sequencing a minuscule $2-3 \%$ of all positive cases, we are still in an ocean of uncertainties to comment on the real number of genuine Omicron cases. ${ }^{4}$ Going by the average consensus worldwide, even if $60-80 \%$ is Omicron, that still implies that Delta and other variants are out there and may retaliate any time. Moreover, we do not know if B1.1.529 is just a transient quasi-species on its evolutionary trajectory in the process of evolving to a more virulent form. We also do not know if Omicron will make us permissive for coinfection by another variant that is perhaps mutating at some corner of the world. The rapid spread of B1.1.529 with its extremely high transmissibility could in theory wipe out COVID-specific memory B/T cells that many of us might be banking on, making them ineffective and futile. Worst, if it blunts the effect of the COVID-19 vaccine, acting as a "decoy virus" exhausting the pool of effector immune cells before another lethal variant steps in. With all these possibilities, labeling Omicron as a harmless variant and taking the guard off is the last thing that one should ever do. Further, the decision for a booster dose needs some serious thoughts following the philosophy that "more is not always the best." Serology testing has shown a lesser efficiency in vaccine-induced neutralization against the new variant (though surely it avoids a severe disease outcome). Under these conditions, there is no credible evidence to show that a third jab will make things all right. We need to consider that sometimes a higher dose of antibodies produced may in fact facilitate virus infection by the process of antibody-dependent enhancement. ${ }^{5}$ Although well observed in Dengue virus infection and not yet in SARS-CoV-2, the probability how-so-ever small still remains. We have already seen a massive breakthrough reinfection among those fully vaccinated making it apparent that the vaccines are not capable enough to prevent new infection or virus transmission. Whether adding a booster dose to ramp the antibody level will do any benefit is highly uncertain and should be only considered based on solid science and rigorous clinical trials, rather than speculation. Furthermore, drawing parallels between $\mathrm{Ab}$ titers with disease, protection cannot only be misleading but also erroneous. ${ }^{6}$ For a country as large and populous as India with just $64 \%$ fully vaccinated with a double dose (as of December 30, 2021), demand for a third booster dose is highly ambitious. Making the right choices and sending the right messages, more so in pandemic times might hold the key to overcoming the ongoing crisis. Sending the wrong message may do more harm than the virus itself. ${ }^{7}$

\section{REFERENCES}

1. Classification of Omicron (B.1.1.529): SARS-CoV-2 Variant of Concern. Available from: https://www.who.int/news/item/26-112021-classification-of-omicron-(b.1.1.529)-sars-cov-2-variantof-concern [Last assessed on 2022 Jan 11].

2. India COVID Cases. Available from: https://www.mohfw.gov.in [Last assessed on $2022 \mathrm{Feb} 5]$

3. It's Dangerous to Take Omicron Lightly. Available from: https:// indianexpress.com/article/opinion/columns/its-dangerous-totake-omicron-lightly-7710469[Last assessed on 2022 Jan 23]

4. Lack of Genome Sequencing Capacity Hampers India's Efforts to Track the Spread of Omicron: Available from: https://scroll.in/ article/1013438/lack-of-genome-sequencing-capacity-hampersindias-efforts-to-track-the-spread-of-omicron [Last assessed 2022 Jan 11].

5. Narayan R and Tripathi S. Intrinsic ADE: The dark side of antibody dependent enhancement during dengue infection. Front Cell Infect Microbiol. 2020;10:580096.

https://doi.org/10.3389/fcimb.2020.580096

6. Bastos ML, Tavaziva G, Abidi SK, Campbell JR, Haraoui LP, Johnston JC, et al. Diagnostic accuracy of serological tests for covid-19: Systematic review and meta-analysis. BMJ. 2020;370:m2516. https://doi.org/10.1136/bmj.m2516

7. https://www.medicallyspeaking.in/experts-speak/sending-thewrong-message/

\section{Authors Contributions:}

All Authors contributed equally towords this review article

Work attributed to:

Department of Biochemistry, All India Institute of Medical Sciences, New Delhi, India and Department of Biochemistry, Jagannath Gupta Institute of Medical Sciences and Hospital, Kolkata, West Bengal, India

\section{ORCID ID:}

Dr. Ruby Dhar - (D https://orcid.org/0000-0003-3600-6554

Dr. Arun Kumar - (1) https://orcid.org/0000-0002-8800-0296

Dr. Subhradip Karmakar - (10) https://orcid.org/0000-0002-4757-8729

Source of Funding: None, Conflicts of Interest: None. 